\title{
Homomorphism Of Tripolar Fuzzy Soft $\Gamma$-Semiring
}

\author{
AHLAM FALLATAH \\ Taibah University \\ Department of Mathematics \\ Madina \\ SAUDI ARABIA
}

\author{
MOURAD OQLA MASSA'DEH \\ Al-Balqa Applied university \\ Department of Applied science \\ Ajloun \\ JORDAN
}

\author{
ABD ULAZEEZ ALKOURI \\ Ajloun National University \\ Department of Mathematics \\ Ajloun \\ JORDAN
}

\begin{abstract}
Given the notion of tripolar fuzzy soft sets, the concepts of a tripolar fuzzy soft $\Gamma-$ Semirings, a tripolar fuzzy soft $\Gamma$-Semiring homomorphism and a tripolar fuzzy soft ideal in $\Gamma$-Semirings are discussed, and related properties and corollaries are investigated. On the other hand, in this paper, we also define the image and pre-image of tripolar fuzzy soft $\Gamma$-Semirings. Some properties and results involving these concepts are stated and proved. .
\end{abstract}

Key Words Soft set, fuzzy Soft set, tripolar fuzzy soft set, tripolar fuzzy soft $\Gamma$-Semiring, tripolar fuzzy soft ideal, tripolar fuzzy soft $\Gamma$-Semiring homomorphism.

Received: October 12, 2019. Revised: May 2, 2020. Accepted: May 11, 2020. Published: May 28, 2020.

\section{Introduction}

In 1934 Vandiver [1] introduced the concept of semiring, a semiring concepet is the best algebraic structure because its comman generalization of distribitive lattices, rings and an universal algebra with two binary operations addition and multiplication such that one of them distributive over the other. Semiring used for solving problems in applied mathematics, information sciences and in the areas of theortical computer science as well as in optimazation theory, coding theory, graph theory and formal languages.

In 1964 Nobusawa [2] gave $\Gamma$-ring notation as a generalization of ring, after that Sen [3] disscused $\Gamma$-semigroup concept. While, $\Gamma$-semiring concept was given by Muirali Krishna [4] as a generalizes to the concept of $\Gamma$-ring and semiring. A fuzzy set theory was discussed and introduced by Zadeh [5] in 1965 as the most appropriate theory for dealing with uncertainty. Rosenfeld [6] in 1971 studied fuzzy subgroup concepts. The idea of fuzzy subgroup and its application on theory and properties studied by some researchers, see [7, 8, 9, 10, 11, 12]. In 1999, the concept of soft set theory was introduced by Molodtsov [13] as a new mathematical tool for dealing with uncertainties. Furthermore, in 2001 Maji et al [14] introduced fuzzy soft sets as extended of soft set theory. Ghosh et al and Murali $[15,16]$ introduced and studied fuzzy soft ring, fuzzy soft ideals and fuzzy soft kideals over a $\Gamma$-Semiring. öztürk et al [17] discussed soft $\Gamma$-rings and fuzzy subnear rings. In 1999, Attanassov [18] gave the idea of intuitionistic fuzzy set.
Massa'deh et al [19, 20, 21, 22, 23] extended the intuitionistic fuzzy set notation to $\Gamma$-Semiring and its ideals, Ku-ideals, subrings, M-subgroups and homomorphisms. On the other hand, Maji et al [24] studied and introduced the intuitionistic fuzzy soft set concept. Then Yaqoob et al [25] studied the concep on groups induced by $(\mathrm{t}, \mathrm{s})$-norm.

In 1998, Zhang [26] introduced bipolar fuzzy sets concepts as a generlazation of fuzzy sets. Lee [27] in 2000 used this concept and applied it to algebraic structures. Also Massa'deh [28, 29, 30] introduced the concepts of bipolar Q-fuzzy H-ideals over $\Gamma$-hemiring, anti bipolar $Q$-fuzzy normal semigroup and bipolar for any cosets, isomorphisms and $\Gamma$-hemiring. Bipolar fuzzy soft set concept introduced in 2013 by Akram [31] where he studied this concept on subalgebras.

The ideas of tripolar fuzzy set was introduced by Murali Krishna Rao [32] in 2018 where he discussed this concept on interior ideal of $\Gamma$-semigroup. Also, Murali et al discussed this concept on interior ideal of $\Gamma$-semiring and on soft interior ideal over semiring [33]. In this paper we introduced and discuss the concept of tripolar fuzzy soft $\Gamma$-semiring homomorphism and some of its theorems and properties of homomorphic image of tripolar fuzzy soft $\Gamma$-semiring.

\section{Preliminaries}

Definition 1. [4] If $S$ is a set together with two associative operations called addition + and multiplica- 
tion then will be called a semiring if the following conditions hold:

1. + is a commutative operation.

2. $\exists 0 \in S$ such that $s+0=s$ and $s \cdot 0=0 \cdot s=$

$0 \forall s \in S$.

3. Distribute low hold from left and right.

Definition 2. [4] If $(S,+)$ and $(\Gamma,+)$ are commutative semigroups. Then $S$ is said to be $\Gamma$-semiring, if there exists a mapping $S \times \Gamma \times S \rightarrow S$ written as $\left(s_{1}, \alpha, s_{2}\right)$ as $s_{1} \alpha s_{2}$ such that it satisfies the following conditions:

1. $s_{1} \alpha\left(s_{2}+s_{3}\right)=s_{1} \alpha s_{2}+s_{1} \alpha s_{3}$

2. $\left(s_{1}+s_{2}\right) \alpha s_{3}=s_{1} \alpha s_{3}+s_{2} \alpha s_{3}$

3. $s_{1}(\alpha+\beta) s_{2}=s_{1} \alpha s_{2}+s_{1} \beta s_{2}$.

4. $s_{1} \alpha\left(s_{2} \beta s_{3}\right)=\left(s_{1} \alpha s_{2}\right) \beta s_{3}, \forall s_{1}, s_{2}, s_{3} \in S$ and $\alpha, \beta \in \Gamma$.

Definition 3. [4] If $M$ is a $\Gamma$-semiring and $I$ be a non empty subset of $M$. Then $I$ is said to be a $\Gamma$-subsemiring of $M$ if $I$ is a sub-semigroup of $(M,+)$ and $I \Gamma I \subseteq I$.

Definition 4. [4] If $M$ is a $\Gamma$-semiring and I is a non empty subset of $M$. Then:

1. I is called a right ideal of $M$ if:

(i) $I$ is closed under addition.

(ii) $I \Gamma M \subseteq I$.

2. I is called a left ideal of $M$ if:

(i) $I$ is closed under addition.

(ii) $M \Gamma I \subseteq I$.

3. I is called an ideal of $M$, if it is both a right and left ideal.

Definition 5. [18] An intuitionistic fuzzy set of a non empty set $A$ is an object of the form $\delta=\left(\delta_{\mu}, \delta_{\lambda}\right)=$ $\left\{\left(a, \delta_{\mu}(a), \delta_{\lambda}(a)\right) ; a \in A\right\}$, such that $\delta_{\mu}: A \rightarrow$ $[0,1], \delta_{\lambda}: A \rightarrow[0,1]$ are membership functions, $\delta_{\mu}, \delta_{\lambda}$ are respectively and $0 \leq \delta_{\mu}(a)+\delta_{\mu}(a) \leq$ $1, \forall a \in A$.

Definition 6. [26] A bipolar fuzzy set $\gamma$ of a non empty set $A$ is an object of the form $\gamma=$ $\left\{\left(a, \gamma_{\mu}(a), \gamma_{\lambda}(a)\right) ; a \in A\right\}$ such that $\gamma_{\mu}: A \rightarrow[0,1]$ and $\gamma_{\lambda}: A \rightarrow[-1,0] \cdot \gamma_{\mu}(a)$ represents satisfaction degree of a to the property corresponding to fuzzy set $\gamma$ and $\gamma_{\lambda}(a)$ represents satisfaction degree of a to the implicit counter property of fuzzy set $\gamma$.

Definition 7. [13] If $U$ is an intial universe set, $E$ is the of parameters set, $X \subset E$. If $P(U)$ represent to the power set of $U$. Then a pair $(\phi, X)$ is said to be a soft set over $U$ such that $\phi$ is a map given by $\phi: X \rightarrow P(u)$.
Definition 8. [14] If $U$ is an intial universe set, $E$ is a parameters set and $X \subseteq E$. A pair $(\phi, X)$ is said to be fuzzy soft over $U$, such that $\phi$ is a map given by $\phi: X \rightarrow I^{U}$ where $I^{U}$ denotes the collection of all fuzzy subset of $U$.

Definition 9. [4] If $R_{1}$ and $R_{2}$ are two $\Gamma$-semirings, a function $\Psi: R_{1} \rightarrow R_{2}$ is called a homomorphism $\Gamma$-semiring if $\Psi(x+y)=\Psi(x)+\Psi(y)$ and $\Psi(x \alpha y)=\Psi(x) \alpha \Psi(y), \forall x, y \in R_{1}, \alpha \in \Gamma$.

Definition 10. [4] If $R_{1}$ and $R_{2}$ are two sets and $\Psi$ : $R_{1} \rightarrow R_{2}$ is any function. A bipolar fuzzy subset $\delta$ of $R_{1}$ is called a $\Psi$-invariant if $\Psi(a)=\Psi(b) \Rightarrow$ $\delta(a)=\delta(b)$.

Definition 11. [30] If $\psi: R_{1} \rightarrow R_{2}$ is a map and $\delta=$ $\left(\delta^{+}, \delta^{-}\right)$and $\gamma=\left(\gamma^{+}, \gamma^{-}\right)$are bipolar fuzzy subset in $R_{1}$ and $R_{2}$ respectively. Then the image $\psi(\delta)$ of $\delta$ is the bipolar fuzzy subset $\psi(\delta)=\left((\psi(\delta))^{+},(\psi(\delta))^{-}\right)$ of $R_{2}$ defined by:

$(\psi(\delta))^{+}(a)=\left\{\begin{array}{l}\max \left\{\delta^{+}(a) ; a \in \psi^{-}(a) ; i f \psi^{-}(a) \neq \phi\right\} \\ 0 ; \text { otherwise }\end{array}\right.$

$(\psi(\delta))^{-}(a)=\left\{\begin{array}{l}\max \left\{\delta^{-}(a) ; a \in \psi^{-}(a) ; i f \psi^{-}(a) \neq \phi\right\} \\ 0 ; \text { otherwise }\end{array}\right.$

and the pre-image $\psi^{-1}(\gamma)$ of $\gamma$ under $\psi$ is the bipolar fuzzy subset of $R_{1}$ defined by for $a \in R_{1},\left(\psi^{-1}(\gamma)\right)^{+}(a)=\gamma^{+}(\psi(a))$ and $\left(\psi^{-1}(\gamma)\right)^{-}(a)=\gamma^{-}(\psi(a))$.

Definition 12. [33] If $Y$ is a universe set, a tripolar fuzzy set $\gamma$ in $Y$ is an object having the form $\gamma=\left\{\left(a, \lambda_{\gamma}(a), \mu_{\gamma}(a), \delta_{\gamma}\right) ; a \in Y\right.$ and $0 \leq \lambda_{\gamma}(a)+$ $\left.\mu_{\gamma}(a) \leq 1\right\}$ such that, $\lambda_{\gamma}: Y \rightarrow[0,1], \mu_{\gamma}: Y \rightarrow$ $[0,1], \delta_{\gamma}: Y \rightarrow[-1,0] ; 0 \leq \lambda_{\gamma}(a)+\mu_{\gamma}(a) \leq 1$. The degree of membership $\lambda_{\gamma}(a)$ characterize the extent that a satisfies the property corresponding to tripolar fuzzy set $\gamma, \mu_{\gamma}(a)$ characterize the extent that a satisfies to the not property corresponding to tripolar fuzzy set $\gamma$ and $\delta_{\gamma}(a)$ characterize the extent that a satisfies to the implicit counter property of tripolar fuzzy set $\gamma$.

Remark 13. $\gamma=\left(\lambda_{\gamma}, \mu_{\gamma}, \delta_{\gamma}\right)$ has been used for $\gamma=\left\{\left(a, \lambda_{\gamma}(a), \mu_{\gamma}(a), \delta_{\gamma}\right) ; a \in Y\right.$ and $0 \leq \lambda_{\gamma}(a)+$ $\left.\mu_{\gamma}(a) \leq 1\right\}$.

Definition 14. [16] Assume that $R$ is a $\Gamma$-semiring, $E$ is a set of parameter and $X \subseteq E$. If $\phi$ is a mapping given by $\phi: X \rightarrow \rho(R)$ such that $\rho(R)$ is the power set of $R$. Then $(\phi, X)$ is called a soft $\Gamma$-semiring over $R$ if and only if for each $x \in X, \phi(x)$ is $\Gamma$-subsemiring of $R$. This means that:

1. $a, b \in R \Rightarrow a+b \in \phi(a)$. 
2. $a, b \in R, \alpha \in \Gamma \Rightarrow a \alpha b \in \phi(a)$.

Definition 15. If $S$ is a $\Gamma$-semiring a tripolar fuzzy soft $(\phi, X)$ over $S$ is said to be tripolar fuzzy soft $\Gamma$-semiring over $S$ if $\phi(x)=$ $\left\{\lambda_{\phi(x)}(s), \mu_{\phi(x)}(s), \delta_{\phi(x)}(s) ; s \in S, x \in X\right\}$ such that $\lambda_{\phi(x)}(s): S \rightarrow[0,1], \mu_{\phi(x)}(s): S \rightarrow$ $[0,1], \delta_{\phi(x)}(s): S \rightarrow[-1,0], 0 \leq \lambda_{\phi(x)}(s)+$ $\mu_{\phi(x)}(s) \leq 1, \forall s \in S$ satisfying the following axioms:

1. $\lambda_{\phi(x)}\left(s_{1}+s_{2}\right) \geq \min \left\{\lambda_{\phi(x)}\left(s_{1}\right), \lambda_{\phi(x)}\left(s_{2}\right)\right\}$

2. $\mu_{\phi(x)}\left(s_{1}+s_{2}\right) \leq \max \left\{\mu_{\phi(x)}\left(s_{1}\right), \mu_{\phi(x)}\left(s_{2}\right)\right\}$

3. $\delta_{\phi(x)}\left(s_{1}+s_{2}\right) \leq \max \left\{\delta_{\phi(x)}\left(s_{1}\right), \delta_{\phi(x)}\left(s_{2}\right)\right\}$

4. $\lambda_{\phi(x)}\left(s_{1} \alpha s_{2}\right) \geq \min \left\{\lambda_{\phi(x)}\left(s_{1}\right), \lambda_{\phi(x)}\left(s_{2}\right)\right\}$

5. $\mu_{\phi(x)}\left(s_{1} \alpha s_{2}\right) \leq \max \left\{\mu_{\phi(x)}\left(s_{1}\right), \mu_{\phi(x)}\left(s_{2}\right)\right\}$

6. $\delta_{\phi(x)}\left(s_{1} \alpha s_{2}\right) \leq \max \left\{\delta_{\phi(x)}\left(s_{1}\right), \delta_{\phi(x)}\left(s_{2}\right)\right\}$, $\forall s_{1}, s_{2} \in S, x \in X$ and $\alpha \in \Gamma$.

Definition 16. [4] If $S$ is a $\Gamma$-semiring, $E$ is a parameter set and $X \subseteq E$. If $\phi$ is a mapping given by $\phi: X \rightarrow \rho(S)$. Then $(\phi, X)$ is said to be a soft right (left) ideal over $S$ if and only if for each $x \in X, \phi(x)$ is a right (left) ideal of $S$. This means that:

1. $s_{1}, s_{2} \in \phi(X)$ then $s_{1}+s_{2} \in \phi(X)$

2. $s_{1}, s_{2} \in \phi(X), \alpha \in \Gamma, s \in S$ then $s_{1} \alpha s\left(s \alpha s_{1}\right) \in$ $\phi(X)$.

Definition 17. A tripolar fuzzy soft set $(\phi, X)$ over $\Gamma$-semiring $S$ is said to be a tripolar fuzzy soft right (left) ideal over $S$ if

1. $\lambda_{\phi(x)}\left(s_{1}+s_{2}\right) \geq \min \left\{\lambda_{\phi(x)}\left(s_{1}\right), \lambda_{\phi(x)}\left(s_{2}\right)\right\}$

2. $\mu_{\phi(x)}\left(s_{1}+s_{2}\right) \leq \max \left\{\mu_{\phi(x)}\left(s_{1}\right), \mu_{\phi(x)}\left(s_{2}\right)\right\}$

3. $\delta_{\phi(x)}\left(s_{1}+s_{2}\right) \leq \max \left\{\delta_{\phi(x)}\left(s_{1}\right), \delta_{\phi(x)}\left(s_{2}\right)\right\}$

4. $\lambda_{\phi(x)}\left(s_{1} \alpha s_{2}\right) \geq \lambda_{\phi(x)}\left(s_{1}\right)\left(\lambda_{\phi(x)}\left(s_{2}\right)\right)$

5. $\mu_{\phi(x)}\left(s_{1} \alpha s_{2}\right) \leq \mu_{\phi(x)}\left(s_{1}\right)\left(\mu_{\phi(x)}\left(s_{2}\right)\right)$

6. $\delta_{\phi(x)}\left(s_{1} \alpha s_{2}\right) \leq \delta_{\phi(x)}\left(s_{1}\right)\left(\delta_{\phi(x)}\left(s_{2}\right)\right), \forall s_{1}, s_{2} \in$ $S, x \in X$ and $\alpha \in \Gamma$.

Definition 18. If $S$ is a $\Gamma$-semiring, $E$ is a parameter set and $X \subseteq E$. A tripolar fuzzy soft set $(\phi, X)$ over $S$ is said to be a tripolar fuzzy soft ideal if the follwing axioms are hold:

1. $\lambda_{\phi(x)}\left(s_{1}+s_{2}\right) \geq \min \left\{\lambda_{\phi(x)}\left(s_{1}\right), \lambda_{\phi(x)}\left(s_{2}\right)\right\}$

2. $\mu_{\phi(x)}\left(s_{1}+s_{2}\right) \leq \max \left\{\mu_{\phi(x)}\left(s_{1}\right), \mu_{\phi(x)}\left(s_{2}\right)\right\}$
3. $\delta_{\phi(x)}\left(s_{1}+s_{2}\right) \leq \max \left\{\delta_{\phi(x)}\left(s_{1}\right), \delta_{\phi(x)}\left(s_{2}\right)\right\}$

4. $\lambda_{\phi(x)}\left(s_{1} \alpha s_{2}\right) \geq \max \left\{\lambda_{\phi(x)}\left(s_{1}\right), \lambda_{\phi(x)}\left(s_{2}\right)\right\}$

5. $\mu_{\phi(x)}\left(s_{1} \alpha s_{2}\right) \leq \min \left\{\mu_{\phi(x)}\left(s_{1}\right), \mu_{\phi(x)}\left(s_{2}\right)\right\}$

6. $\delta_{\phi(x)}\left(s_{1} \alpha s_{2}\right) \leq \min \left\{\delta_{\phi(x)}\left(s_{1}\right), \delta_{\phi(x)}\left(s_{2}\right)\right\}$, $\forall s_{1}, s_{2} \in S, x \in X$ and $\alpha \in \Gamma$.

\section{Homomorphism in tripolar fuzzy soft $\Gamma$-semiring}

The homomorphism concept over tripolar fuzzy soft $\Gamma$-semiring is introduced and studied their properties in this section.

Definition 19. If $\left(\phi_{1}, X\right)$ and $\left(\phi_{2}, Y\right)$ are tripolar fuzzy soft set over $\Gamma$-semirings $R_{1}$ and $R_{2}$ respectiely. Let $\psi_{1}: R_{1} \rightarrow R_{2}$ and $\psi_{2}: X \rightarrow Y$ are two functions such that $X$ and $Y$ are parameter sets for the crisp sets $R_{1}$ and $R_{2}$ respectiely. Then $\left(\psi_{1}, \psi_{2}\right)$ is said to be a tripolar fuzzy soft function from $R_{1}$ to $R_{2}$.

Definition 20. If $\left(\phi_{1}, X\right)$ and $\left(\phi_{2}, Y\right)$ are tripolar fuzzy soft set over $\Gamma$-semirings $R_{1}$ and $R_{2}$ respectiely and $\left(\psi_{1}, \psi_{2}\right)$ are tripolar fuzzy soft functions from $R_{1}$ to $R_{2}$. Then $\left(\psi_{1}, \psi_{2}\right)$ is called tripolar fuzzy soft $\Gamma$-semiring homomorphism if satisfying the following axioms:

1. $\psi_{1}$ is a $\Gamma$-semiring homomorphism from $R_{1}$ onto $R_{2}$.

2. $\psi_{2}$ is a mapping from $X$ onto $Y$.

3. $\psi_{1}\left(\lambda_{\phi_{1}(x)}\right)=\phi_{2 \psi_{2}(x)}, \psi_{1}\left(\mu_{\phi_{1}(x)}\right)=\phi_{2 \psi_{2}(x)}$ and $\psi_{1}\left(\delta_{\phi_{1}(x)}\right)=\phi_{2 \psi_{2}(x)}, \forall x \in X$.

Remark 21. If there exist a tripolar fuzzy soft $\Gamma$-semiring homomorphism between $\left(\phi_{1}, X\right)$ and $\left(\phi_{2}, Y\right)$. Then we say that $\left(\phi_{1}, X\right)$ is soft homomorphic to $\left(\phi_{2}, Y\right)$.

Definition 22. If $\left(\psi_{1}, \psi_{2}\right)$ is a tripolar fuzzy soft function from $R_{1}$ to $R_{2}$. The pre-image of $\left(\phi_{2}, Y\right)$ under the tripolar fuzzy soft function $\left(\psi_{1}, \psi_{2}\right)$, denoted by $\left(\psi_{1}, \psi_{2}\right)^{-1}\left(\left(\phi_{2}, Y\right)\right.$ defined by $\left(\psi_{1}, \psi_{2}\right)^{-1}\left(\phi_{2}, Y\right)=$ $\left(\psi_{1}^{-1}\left(\phi_{2}\right), \psi_{2}^{-1}(Y)\right)$ is a tripolar fuzzy soft set.

Theorem 23. If $(\phi, X)$ is a tripolar fuzzy soft $\Gamma$-semiring over $R_{2}, \psi: R_{1} \rightarrow R_{2}$ is monomorphism and for each $x \in X$, define $(\psi \phi)_{x}(r)=$ $\phi_{x}(\psi(r)), \forall r \in R$, then $(\psi \phi, X)$ is a tripolar fuzzy soft $\Gamma$-semiring over $R_{2}$. 
Proof. Let $r_{1}, r_{2} \in R, x \in X$ and $\alpha \in \Gamma$. Then:

$$
\begin{aligned}
& \text { 1. }(\psi \phi)_{x}\left(r_{1}+r_{2}\right)=\phi_{x}\left(\psi\left(r_{1}+r_{2}\right)\right) \\
& =\lambda_{\phi(x)}\left(\psi\left(r_{1}\right)+\psi\left(r_{2}\right)\right) \\
& \geq \min \left\{\lambda_{\phi(x)}\left(\psi\left(r_{1}\right)\right), \lambda_{\phi(x)}\left(\psi\left(r_{2}\right)\right)\right\} \\
& =\min \left\{(\psi \phi)_{x}\left(r_{1}\right),(\psi \phi)_{x}\left(r_{2}\right)\right\} . \\
& 2 .(\psi \phi)_{x}\left(r_{1}+r_{2}\right)=\phi_{x}\left(\psi\left(r_{1}+r_{2}\right)\right) \\
& =\mu_{\phi(x)}\left(\psi\left(r_{1}\right)+\psi\left(r_{2}\right)\right) \\
& \leq \max \left\{\mu_{\phi(x)}\left(\psi\left(r_{1}\right)\right), \mu_{\phi(x)}\left(\psi\left(r_{2}\right)\right)\right\} \\
& =\max \left\{(\psi \phi)_{x}\left(r_{1}\right),(\psi \phi)_{x}\left(r_{2}\right)\right\} . \\
& 3 .(\psi \phi)_{x}\left(r_{1}+r_{2}\right)=\phi_{x}\left(\psi\left(r_{1}+r_{2}\right)\right) \\
& =\delta_{\phi(x)}\left(\psi\left(r_{1}\right)+\psi\left(r_{2}\right)\right) \\
& \leq \max \left\{\delta_{\phi(x)}\left(\psi\left(r_{1}\right)\right), \delta_{\phi(x)}\left(\psi\left(r_{2}\right)\right)\right\} \\
& =\max \left\{(\psi \phi)_{x}\left(r_{1}\right),(\psi \phi)_{x}\left(r_{2}\right)\right\} . \\
& 4 .(\psi \phi)_{x}\left(r_{1} \alpha r_{2}\right)=\phi_{x}\left(\psi\left(r_{1} \aleph r_{2}\right)\right) \\
& =\lambda_{\phi(x)}\left(\psi\left(r_{1}\right) \alpha \psi\left(r_{2}\right)\right) \\
& \geq \min \left\{\lambda_{\phi(x)}\left(\psi\left(r_{1}\right)\right), \lambda_{\phi(x)}\left(\psi\left(r_{2}\right)\right)\right\} \\
& =\min \left\{(\psi \phi)_{x}\left(r_{1}\right),(\psi \phi)_{x}\left(r_{2}\right)\right\} . \\
& 5 .(\psi \phi)_{x}\left(r_{1} \alpha r_{2}\right)=\phi_{x}\left(\psi\left(r_{1} \alpha r_{2}\right)\right) \\
& =\mu_{\phi(x)}\left(\psi\left(r_{1}\right) \alpha \psi\left(r_{2}\right)\right) \\
& \leq \max \left\{\mu_{\phi(x)}\left(\psi\left(r_{1}\right)\right), \mu_{\phi(x)}\left(\psi\left(r_{2}\right)\right)\right\} \\
& =\max \left\{(\psi \phi)_{x}\left(r_{1}\right),(\psi \phi)_{x}\left(r_{2}\right)\right\} . \\
& 6 .(\psi \phi)_{x}\left(r_{1} \alpha r_{2}\right) \\
& =\phi_{x}\left(\psi\left(r_{1} \alpha r_{2}\right)\right) \\
& =\delta_{\phi(x)}\left(\psi\left(r_{1}\right) \alpha \psi\left(r_{2}\right)\right) \\
& \leq \max \left\{\delta_{\phi(x)}\left(\psi\left(r_{1}\right)\right), \delta_{\phi(x)}\left(\psi\left(r_{2}\right)\right)\right\} \\
& =\max \left\{(\psi \phi)_{x}\left(r_{1}\right),(\psi \phi)_{x}\left(r_{2}\right)\right\} . \\
&
\end{aligned}
$$

Therefore $(\psi \phi)_{x}$ is a tripolar fuzzy $\Gamma$-subsemiring of $S$. Thus $(\psi f, X)$ is a tripolar fuzzy soft $\Gamma$-semiring over $R_{2}$.

Theorem 24. If $(\gamma, X)$ is a tripolar fuzzy soft semiring over $\Gamma$-semiring $R$, if $\psi$ is an endomomorphism of $R$ and defined $(\gamma \psi)_{x}=\gamma_{x} \psi$ for each $x \in X$. Then $(\gamma \psi, X)$ is a tripolar fuzzy soft $\Gamma$-semiring over $R$.

Proof. Let $r_{1}, r_{2} \in R, x \in X$ and $\alpha \in \Gamma$. Then:

$$
\begin{aligned}
& \text { 1. }(\lambda \psi)_{x}\left(r_{1}+r_{2}\right) \\
& =\lambda_{\gamma(x)}\left(\psi\left(r_{1}+r_{2}\right)\right) \\
& =\lambda_{\gamma(x)}\left(\psi\left(r_{1}\right)+\psi\left(r_{2}\right)\right) \\
& \geq \min \left\{\lambda_{\gamma(x)}\left(\psi\left(r_{1}\right)\right), \lambda_{\gamma(x)}\left(\psi\left(r_{2}\right)\right)\right\} \\
& =\min \left\{(\lambda \psi)_{x}\left(r_{1}\right),(\lambda \psi)_{x}\left(r_{2}\right)\right\} . \\
& \text { 2. }(\mu \psi)_{x}\left(r_{1}+r_{2}\right) \\
& =\mu_{\gamma(x)}\left(\psi\left(r_{1}+r_{2}\right)\right) \\
& =\mu_{\gamma(x)}\left(\psi\left(r_{1}\right)+\psi\left(r_{2}\right)\right) \\
& \leq \max \left\{\mu_{\gamma(x)}\left(\psi\left(r_{1}\right)\right), \mu_{\gamma(x)}\left(\psi\left(r_{2}\right)\right)\right\} \\
& =\max \left\{(\mu \psi)_{x}\left(r_{1}\right),(\mu \psi)_{x}\left(r_{2}\right)\right\} . \\
& 3 .(\delta \psi)_{x}\left(r_{1}+r_{2}\right) \\
& =\delta_{\gamma(x)}\left(\psi\left(r_{1}+r_{2}\right)\right) \\
& =\delta_{\gamma(x)}\left(\psi\left(r_{1}\right)+\psi\left(r_{2}\right)\right) \\
& \leq \max \left\{\delta_{\gamma(x)}\left(\psi\left(r_{1}\right)\right), \delta_{\gamma(x)}\left(\psi\left(r_{2}\right)\right)\right\} \\
& =\max \left\{(\delta \psi)_{x}\left(r_{1}\right),(\delta \psi)_{x}\left(r_{2}\right)\right\} .
\end{aligned}
$$

$$
\begin{aligned}
& \text { 4. }(\lambda \psi)_{x}\left(r_{1} \alpha r_{2}\right) \\
& =\lambda_{\gamma(x)}\left(\psi\left(r_{1} \aleph r_{2}\right)\right) \\
& =\lambda_{\gamma(x)}\left(\psi\left(r_{1}\right) \alpha \psi\left(r_{2}\right)\right) \\
& \geq \min \left\{\lambda_{\gamma(x)}\left(\psi\left(r_{1}\right)\right), \lambda_{\gamma(x)}\left(\psi\left(r_{2}\right)\right)\right\} \\
& =\min \left\{(\lambda \psi)_{x}\left(r_{1}\right),(\lambda \psi)_{x}\left(r_{2}\right)\right\} . \\
& \text { 5. }(\mu \psi)_{x}\left(r_{1} \alpha r_{2}\right) \\
& =\mu_{\gamma(x)}\left(\psi\left(r_{1} \alpha r_{2}\right)\right) \\
& =\mu_{\gamma(x)}\left(\psi\left(r_{1}\right) \alpha \psi\left(r_{2}\right)\right) \\
& \leq \max \left\{\mu_{\gamma(x)}\left(\psi\left(r_{1}\right)\right), \mu_{\gamma(x)}\left(\psi\left(r_{2}\right)\right)\right\} \\
& =\max \left\{(\mu \psi)_{x}\left(r_{1}\right),(\mu \psi)_{x}\left(r_{2}\right)\right\} . \\
& 6 .(\delta \psi)_{x}\left(r_{1} \alpha r_{2}\right) \\
& =\delta_{\gamma(x)}\left(\psi\left(r_{1} \alpha r_{2}\right)\right) \\
& =\delta_{\gamma(x)}\left(\psi\left(r_{1}\right) \alpha \psi\left(r_{2}\right)\right) \\
& \leq \max \left\{\delta_{\gamma(x)}\left(\psi\left(r_{1}\right)\right), \delta_{\gamma(x)}\left(\psi\left(r_{2}\right)\right)\right\} \\
& =\max \left\{(\delta \psi)_{x}\left(r_{1}\right),(\delta \psi)_{x}\left(r_{2}\right)\right\} .
\end{aligned}
$$

Thus $(\gamma \psi)_{x}$ is a tripolar fuzzy $\Gamma$-subsemiring of $R$. Then $(\gamma \psi, X)$ is a tripolar fuzzy soft $\Gamma$-semiring over $R$

Theorem 25. If $\psi: R_{1} \rightarrow R_{2}$ is an eipomomorphism of $\Gamma$-semiring and $(\gamma, X)$ is a tripolar fuzzy soft right ideal over $R_{2}$. If for each $x \in X, \zeta_{x}=\psi^{-1}\left(\gamma_{x}\right)$ then $(\zeta, X)$ is a tripolar fuzzy soft right ideal over $R_{1}$.

Proof. If $x \in X$ and $\alpha \in \Gamma$. Then $\gamma_{x}$ is a tripolar fuzzy soft right ideal over $R_{2}$. If $r_{1}, r_{2} \in R_{1}$ and $\alpha \in \Gamma$, then:

$$
\begin{aligned}
& \text { 1. } \psi^{-1}\left(\lambda_{x}\right)\left(r_{1}+r_{2}\right)=\lambda_{\gamma(x)}\left(\psi\left(r_{1}+r_{2}\right)\right) \\
& =\lambda_{\gamma(x)}\left(\psi\left(r_{1}\right)+\psi\left(r_{2}\right)\right) \\
& \geq \min \left\{\lambda_{\gamma(x)}\left(\psi\left(r_{1}\right)\right), \lambda_{\gamma(x)}\left(\psi\left(r_{2}\right)\right)\right\} \\
& =\min \left\{\psi^{-1}\left(\lambda_{x}\right)\left(r_{1}\right), \psi^{-1}\left(\lambda_{x}\right)\left(r_{2}\right)\right\} \text {. } \\
& 2 . \psi^{-1}\left(\mu_{x}\right)\left(r_{1}+r_{2}\right)=\mu_{\gamma(x)}\left(\psi\left(r_{1}+r_{2}\right)\right) \\
& =\mu_{\gamma(x)}\left(\psi\left(r_{1}\right)+\psi\left(r_{2}\right)\right) \\
& \leq \max \left\{\mu_{\gamma(x)}\left(\psi\left(r_{1}\right)\right), \mu_{\gamma(x)}\left(\psi\left(r_{2}\right)\right)\right\} \\
& =\max \left\{\psi^{-1}\left(\mu_{x}\right)\left(r_{1}\right), \psi^{-1}\left(\mu_{x}\right)\left(r_{2}\right)\right\} \text {. } \\
& 3 . \psi^{-1}\left(\delta_{x}\right)\left(r_{1}+r_{2}\right)=\delta_{\gamma(x)}\left(\psi\left(r_{1}+r_{2}\right)\right) \\
& =\delta_{\gamma(x)}\left(\psi\left(r_{1}\right)+\psi\left(r_{2}\right)\right) \\
& \leq \max \left\{\delta_{\gamma(x)}\left(\psi\left(r_{1}\right)\right), \delta_{\gamma(x)}\left(\psi\left(r_{2}\right)\right)\right\} \\
& =\max \left\{\psi^{-1}\left(\delta_{x}\right)\left(r_{1}\right), \psi^{-1}\left(\delta_{x}\right)\left(r_{2}\right)\right\} \text {. } \\
& \text { 4. } \psi^{-1}\left(\lambda_{x}\right)\left(r_{1} \alpha r_{2}\right)=\lambda_{x}\left(\psi\left(r_{1} \alpha r_{2}\right)\right) \\
& =\lambda_{x}\left(\psi\left(r_{1}\right) \alpha \psi\left(r_{2}\right)\right) \\
& \geq \lambda_{x}\left(\psi\left(r_{1}\right)\right) \\
& =\psi^{-1}\left(\lambda_{x}\right)\left(r_{1}\right) \text {. } \\
& 5 . \psi^{-1}\left(\mu_{x}\right)\left(r_{1} \alpha r_{2}\right)=\mu_{x}\left(\psi\left(r_{1} \alpha r_{2}\right)\right) \\
& =\mu_{x}\left(\psi\left(r_{1}\right) \alpha \psi\left(r_{2}\right)\right) \\
& \leq \mu_{x}\left(\psi\left(r_{1}\right)\right) \\
& =\psi^{-1}\left(\mu_{x}\right)\left(r_{1}\right) \text {. } \\
& 6 . \psi^{-1}\left(\delta_{x}\right)\left(r_{1} \alpha r_{2}\right)=\delta_{x}\left(\psi\left(r_{1} \alpha r_{2}\right)\right) \\
& =\delta_{x}\left(\psi\left(r_{1}\right) \alpha \psi\left(r_{2}\right)\right) \\
& \leq \delta_{x}\left(\psi\left(r_{1}\right)\right) \\
& =\psi^{-1}\left(\delta_{x}\right)\left(r_{1}\right) \text {. }
\end{aligned}
$$


Therefore $\zeta_{x}=\psi^{-1}\left(\gamma_{x}\right)$ is a tripolar fuzzy right ideal of $R_{1}$. Thus $(\zeta, X)$ is a tripolar fuzzy soft right ideal over $R_{1}$. ideal.

Theorem 25 is true for tripolar fuzzy soft left

Proposition 26. If $R_{1}$ and $R_{2}$ are $\Gamma$-semirings, $\psi$ : $R_{1} \rightarrow R_{2}$ is a $\Gamma$-semiring homomorphism and $\phi$ is a $\psi$-invariant bipolar fuzzy subset of $R_{1}$, if $b=\psi(a)$ then $\psi(\phi)(b)=\phi(a) ; a \in R_{1}$.

Proof. strightforword.

Theorem 27. If $(\gamma, X)$ is a tripolar fuzzy soft right ideal over $\Gamma$-semiring $R_{1}$ and $\psi$ is a homomorphism from $R_{1}$ onto $R_{2}$. For each $x \in X, \gamma_{x}$ is a $\psi$-invariant bipolar fuzzy right ideal of $R_{1}$, if $\zeta_{x}=$ $\psi\left(\gamma_{x}\right)$ then $(\zeta, X)$ is a tripolar fuzzy soft right ideal over $R_{2}$.

Proof. Let $r_{1}, r_{2} \in R_{2}, x \in X$ and $\alpha \in \Gamma$. Then there exists $r_{3}, r_{4} \in R_{1}$ such that $\psi\left(r_{2}\right)=r_{1}, \psi\left(r_{4}\right)=$ $r_{2}, r_{1}+r_{2}=\psi\left(r_{3}+r_{4}\right)$ and $r_{1} \alpha r_{2}=\psi\left(r_{3} \alpha r_{4}\right) \cdot \gamma_{x}$ is $\psi$-invariant. Thus by propostion 26 , we have:

$$
\begin{aligned}
1 . \lambda_{\zeta(x)}\left(r_{1}+r_{2}\right) & =\psi\left(\lambda_{\gamma(x)}\right)\left(r_{1}+r_{2}\right) \\
& =\lambda_{\gamma(x)}\left(r_{3}+r_{4}\right) \\
& \geq \min \left\{\lambda_{\gamma(x)}\left(r_{3}\right), \lambda_{\gamma(x)}\left(r_{4}\right)\right\} \\
& =\min \left\{\psi \left(\lambda_{\gamma(x)}\left(r_{1}\right), \psi\left(\lambda_{\gamma(x)}\left(r_{2}\right)\right\}\right.\right. \\
& =\min \left\{\lambda_{\zeta(x)}\left(r_{1}\right), \lambda_{\zeta(x)}\left(r_{2}\right)\right\} \\
2 . \mu_{\zeta(x)}\left(r_{1}+r_{2}\right) & =\psi\left(\mu_{\gamma(x)}\right)\left(r_{1}+r_{2}\right) \\
& =\mu_{\gamma(x)}\left(r_{3}+r_{4}\right) \\
& \leq \max \left\{\mu_{\gamma(x)}\left(r_{3}\right), \mu_{\gamma(x)}\left(r_{4}\right)\right\} \\
& =\max \left\{\psi\left(\mu_{\gamma(x)}\right)\left(r_{1}\right), \psi\left(\mu_{\gamma(x)}\left(r_{2}\right)\right)\right\} \\
& =\max \left\{\mu_{\zeta(x)}\left(r_{1}\right), \mu_{\zeta(x)}\left(r_{2}\right)\right\} \\
3 . \delta_{\zeta(x)}\left(r_{1}+r_{2}\right) & =\psi\left(\delta_{\gamma(x)}\right)\left(r_{1}+r_{2}\right) \\
& =\delta_{\gamma(x)}\left(r_{3}+r_{4}\right) \\
& \leq \max \left\{\delta_{\gamma(x)}\left(r_{3}\right), \delta_{\gamma(x)}\left(r_{4}\right)\right\} \\
& =\max \left\{\psi\left(\delta_{\gamma(x)}\left(r_{1}\right)\right), \psi\left(\delta_{\gamma(x)}\left(r_{2}\right)\right)\right\} \\
& =\max \left\{\delta_{\zeta(x)}\left(r_{1}\right), \delta_{\zeta(x)}\left(r_{2}\right)\right\} \\
& =\psi\left(\lambda_{\gamma(x)}\right)\left(r_{1} \alpha r_{2}\right) \\
& =\lambda_{\gamma(x)}\left(\psi\left(r_{3} \alpha r_{2}\right)\right) \\
& =\lambda_{\gamma(x)}\left(\psi\left(r_{3}\right) \alpha \psi\left(r_{4}\right)\right) \\
& \geq \lambda_{\gamma(x)}\left(\psi\left(r_{3}\right)\right) \\
& =\psi\left(\lambda_{\gamma(x)}\left(r_{1}\right)\right) \\
& =\lambda_{\zeta(x)}\left(r_{1}\right) \\
& =\psi\left(\mu_{\gamma(x)}\right)\left(r_{1} \alpha r_{2}\right) \\
& =\mu_{\gamma(x)}\left(\psi\left(r_{3} \alpha r_{2}\right)\right) \\
& =\mu_{\gamma(x)}\left(\psi\left(r_{3}\right) \alpha \psi\left(r_{4}\right)\right) \\
\leq & \mu_{\gamma(x)}\left(\psi\left(r_{3}\right)\right) \\
& =\psi\left(\mu_{\gamma(x)}\left(r_{1}\right)\right) \\
& =\mu_{\zeta(x)}\left(r_{1}\right) \\
5 . \mu_{\zeta(x)}\left(r_{1} \alpha r_{2}\right) &
\end{aligned}
$$

$$
\begin{aligned}
6 . \delta_{\zeta(x)}\left(r_{1} \alpha r_{2}\right) & =\psi\left(\delta_{\gamma(x)}\right)\left(r_{1} \alpha r_{2}\right) \\
& =\delta_{\gamma(x)}\left(\psi\left(r_{3} \alpha r_{2}\right)\right) \\
& =\delta_{\gamma(x)}\left(\psi\left(r_{3}\right) \alpha \psi\left(r_{4}\right)\right) \\
& \leq \delta_{\gamma(x)}\left(\psi\left(r_{3}\right)\right) \\
& =\psi\left(\delta_{\gamma(x)}\left(r_{1}\right)\right) \\
& =\delta_{\zeta(x)}\left(r_{1}\right)
\end{aligned}
$$

then $\zeta_{x}$ is a tripolar fuzzy ideal of $R_{2}$. Hence $(\zeta, X)$ is a tripolar fuzzy soft right ideal over $R_{2}$.

Theorem 28. If $\left(\gamma_{1}, X_{1}\right)$ and $\left(\gamma_{2}, X_{2}\right)$ are two bipolar fuzzy soft $\Gamma$-semirings over $R_{1}$ and $R_{2}$ respectively, and $(\phi, \psi)$ is a tripolar fuzzy soft $\Gamma$-semiring homomorphism from $\left(\gamma_{1}, X_{1}\right)$ onto $\left(\gamma_{2}, X_{2}\right)$. Then $\left(\phi\left(\gamma_{1}\right), X_{2}\right)$ is a tripolar fuzzy soft $\Gamma$-semiring over $R_{2}$.

Proof. By definition 20, $\phi$ is a $\Gamma$-semiring homomorphism from $R_{1}$ onto $R_{2}$ and $\psi$ is a mapping from $X_{1}$ onto $X_{2}$ for each $y \in X_{2}$ there exist $x \in X$; such that $\psi(x)=y$. Define $\left(\phi\left(\gamma_{1}\right)\right)_{y}=\phi\left(\gamma_{1 x}\right)$. If $r_{1}, r_{2} \in R_{2}$ and $\alpha \in \Gamma$, then there exist $r_{3}, r_{4} \in R_{1}$ such that $\phi\left(r_{3}\right)=r_{1}, \phi\left(r_{4}\right)=r_{2}$ and $\phi\left(r_{3}+r_{4}\right)=r_{1}+r_{2}$ and $\phi\left(r_{3} \alpha r_{4}\right)=r_{1} \alpha r_{2}$. Thus we have:

$$
\begin{aligned}
& \text { 1. }\left(\phi\left(\lambda_{\gamma_{1}}\right)_{\psi(x)}\left(r_{1}+r_{2}\right)=\phi\left(\lambda_{\gamma_{1}}(x)\right)\left(r_{1}+r_{2}\right)\right. \\
& =\lambda_{\gamma_{1}}(x)\left(r_{3}+r_{4}\right) \\
& \geq \min \left\{\lambda_{\gamma_{1}}(x)\left(r_{3}\right), \lambda_{\gamma_{1}}(x)\left(r_{4}\right)\right\} \\
& =\min \left\{\phi\left(\lambda_{\gamma_{1}}(x)\right)\left(r_{1}\right), \phi\left(\lambda_{\gamma_{1}}(x)\right)\left(r_{2}\right)\right\} \\
& =\min \left\{\phi\left(\lambda_{\gamma_{1}}\right)_{\psi(x)}\left(r_{1}\right), \phi\left(\lambda_{\gamma_{1}}\right)_{\psi(x)}\left(r_{2}\right)\right\} \\
& \text { 2.( }\left(\phi\left(\mu_{\gamma_{1}}\right)_{\psi(x)}\left(r_{1}+r_{2}\right)=\phi\left(\mu_{\gamma_{1}}(x)\right)\left(r_{1}+r_{2}\right)\right. \\
& =\mu_{\gamma_{1}}(x)\left(r_{3}+r_{4}\right) \\
& \leq \max \left\{\mu_{\gamma_{1}}(x)\left(r_{3}\right), \mu_{\gamma_{1}}(x)\left(r_{4}\right)\right\} \\
& =\max \left\{\phi\left(\mu_{\gamma_{1}}(x)\right)\left(r_{1}\right), \phi\left(\mu_{\gamma_{1}}(x)\right)\left(r_{2}\right)\right\} \\
& =\max \left\{\phi\left(\mu_{\gamma_{1}}\right)_{\psi(x)}\left(r_{1}\right), \phi\left(\mu_{\gamma_{1}}\right)_{\psi(x)}\left(r_{2}\right)\right\} \\
& \text { 3. }\left(\phi\left(\delta_{\gamma_{1}}\right)_{\psi(x)}\left(r_{1}+r_{2}\right)=\phi\left(\delta_{\gamma_{1}}(x)\right)\left(r_{1}+r_{2}\right)\right. \\
& =\delta_{\gamma_{1}}(x)\left(r_{3}+r_{4}\right) \\
& \leq \max \left\{\delta_{\gamma_{1}}(x)\left(r_{3}\right), \delta_{\gamma_{1}}(x)\left(r_{4}\right)\right\} \\
& =\max \left\{\phi\left(\delta_{\gamma_{1}}(x)\right)\left(r_{1}\right), \phi\left(\delta_{\gamma_{1}}(x)\right)\left(r_{2}\right)\right\} \\
& =\max \left\{\phi\left(\delta_{\gamma_{1}}\right)_{\psi(x)}\left(r_{1}\right), \phi\left(\delta_{\gamma_{1}}\right)_{\psi(x)}\left(r_{2}\right)\right\} \\
& \text { 4. }\left(\phi\left(\lambda_{\gamma_{1}}\right)\right)_{\psi(x)}\left(r_{1} \alpha r_{2}\right)=\phi\left(\lambda_{\gamma_{1}(x)}\right)\left(r_{1} \alpha r_{2}\right) \\
& =\lambda_{\gamma_{1}(x)}\left(r_{3} \alpha r_{4}\right) \\
& \geq \min \left\{\lambda_{\gamma_{1}(x)}\left(r_{3}\right), \lambda_{\gamma_{1}(x)}\left(r_{4}\right)\right\} \\
& =\min \left\{\phi\left(\lambda_{\gamma_{1}(x)}\right)\left(r_{1}\right), \phi\left(\lambda_{\gamma_{1}(x)}\right)\left(r_{2}\right)\right\} \\
& =\min \left\{\phi\left(\lambda_{\gamma_{1}}\right)_{\psi(x)}\left(r_{1}\right), \phi\left(\lambda_{\gamma_{1}}\right)_{\psi(x)}\left(r_{2}\right)\right\} \\
& 5 .\left(\phi\left(\mu_{\gamma_{1}}\right)\right)_{\psi(x)}\left(r_{1} \alpha r_{2}\right)=\phi\left(\mu_{\gamma_{1}(x)}\right)\left(r_{1} \alpha r_{2}\right) \\
& =\mu_{\gamma_{1}(x)}\left(r_{3} \alpha r_{4}\right) \\
& \leq \max \left\{\mu_{\gamma_{1}(x)}\left(r_{3}\right), \mu_{\gamma_{1}(x)}\left(r_{4}\right)\right\} \\
& =\max \left\{\phi\left(\mu_{\gamma_{1}(x)}\right)\left(r_{1}\right), \phi\left(\mu_{\gamma_{1}(x)}\right)\left(r_{2}\right)\right\} \\
& =\max \left\{\phi\left(\mu_{\gamma_{1}}\right)_{\psi(x)}\left(r_{1}\right), \phi\left(\mu_{\gamma_{1}}\right)_{\psi(x)}\left(r_{2}\right)\right\} \\
&
\end{aligned}
$$




$$
\begin{aligned}
& \text { 6. }\left(\phi\left(\delta_{\gamma_{1}}\right)\right)_{\psi(x)}\left(r_{1} \alpha r_{2}\right)=\phi\left(\delta_{\gamma_{1}(x)}\right)\left(r_{1} \alpha r_{2}\right) \\
& =\delta_{\gamma_{1}(x)}\left(r_{3} \alpha r_{4}\right) \\
& \leq \max \left\{\delta_{\gamma_{1}(x)}\left(r_{3}\right), \delta_{\gamma_{1}(x)}\left(r_{4}\right)\right\} \\
& =\max \left\{\phi\left(\delta_{\gamma_{1}(x)}\right)\left(r_{1}\right), \phi\left(\delta_{\gamma_{1}(x)}\right)\left(r_{2}\right)\right\} \\
& =\max \left\{\phi\left(\delta_{\gamma_{1}}\right)_{\psi(x)}\left(r_{1}\right), \phi\left(\delta_{\gamma_{1}}\right)_{\psi(x)}\left(r_{2}\right)\right\} .
\end{aligned}
$$

Then $\phi\left(\gamma_{1}\right)_{y}$ is a tripolar fuzzy $\Gamma$-subsemiring of $R_{2}$. Hence $\left(\phi\left(\gamma_{1}\right), X_{2}\right)$ is a tripolar fuzzy soft $\Gamma$-semiring over $R_{2}$.

Theorem 29. If $R_{1}, R_{2}$ are two $\Gamma$-semirings, $\phi$ : $R_{1} \rightarrow R_{2}$ is a $\Gamma$-semiring homomorphism, $\left(\gamma_{1}, X_{1}\right),\left(\gamma_{2}, X_{2}\right)$ are tripolar fuzzy soft $\Gamma$-semirings over $R_{1}$ and $\left(\gamma_{1}, X_{1}\right)$ is a tripolar fuzzy soft $\Gamma$-subsemiring of $\left(\gamma_{2}, X_{2}\right)$. Then $\left(\phi\left(\gamma_{1}\right), X_{1}\right)$ and $\left(\phi\left(\gamma_{2}\right), X_{2}\right)$ are tripolar fuzzy soft $\Gamma$-subsemirings over $R_{2}$ and $\left(\phi\left(\gamma_{1}\right), X_{1}\right)$ is a tripolar fuzzy soft $\Gamma$-subsemiring of $\left(\phi\left(\gamma_{2}\right), X_{2}\right)$.

Proof. Since $\left(\phi\left(\gamma_{1}\right)\right)_{x}=\phi\left(\gamma_{1(x)}\right)$ is a tripolar fuzzy $\Gamma$-subsemiring of $R_{2}$ for all $x \in X_{1}$ and $\left(\phi\left(\gamma_{2}\right)\right)_{y}=$ $\phi\left(\gamma_{2(y)}\right)$ is a tripolar fuzzy $\Gamma$-subsemiring of $R_{2}$ for all $y \in X_{2}$. Hence $\left(\phi\left(\gamma_{1}\right), X_{1}\right)$ and $\left(\phi\left(\gamma_{2}\right), X_{2}\right)$ are tripolar fuzzy soft $\Gamma$-semiring over $R_{2}$. Since $\left(\gamma_{1}, X_{1}\right)$ is a tripolar fuzzy soft $\Gamma$-subsemiring of $\left(\gamma_{2}, X_{2}\right)$. And $\gamma_{1(x)}$ is a tripolar fuzzy subsemiring of $\gamma_{2(x)}$. Hence $\phi\left(\gamma_{1(x)}\right)$ is a tripolar fuzzy $\Gamma$-subsemiring of $\phi\left(\gamma_{2(x)}\right)$ for all $x \in X_{1}$. Therefore $\left(\phi\left(\gamma_{1}\right), X_{1}\right)$ is a tripolar fuzzy soft $\Gamma$-subsemiring of $\left(\phi\left(\gamma_{2}\right), X_{2}\right)$.

Theorem 30. If $\left(\gamma_{1}, X\right)$ and $\left(\gamma_{2}, Y\right)$ are tripolar fuzzy soft $\Gamma$-semirings over $R_{1}$ and $R_{2}$ respectively and $(\phi, \psi)$ is a tripolar fuzzy soft homomorphism from $\left(\gamma_{1}, X\right)$ onto $\left(\gamma_{2}, Y\right)$ then the pre-image of $\left(\gamma_{2}, Y\right)$ under tripolar fuzzy soft $\Gamma$-semiring homomorphism is a tripolar fuzzy soft $\Gamma$-subsemiring of $\left(\gamma_{1}, X\right)$ over $R_{1}$.

Proof. By Definition $22(\phi, \psi)^{-1}\left(\gamma_{2}, Y\right)=$ $\left(\phi^{-1}\left(\gamma_{2}\right), \psi^{-1}(Y)\right)$. Define $\left(\phi^{-1}\left(\gamma_{2}\right)\right)_{x}\left(r_{1}\right)=$ $\gamma_{2 \psi(x)}\left(\phi\left(r_{1}\right)\right)$ for all $r_{1} \in R_{1}$ and $x \in \psi^{-1}(Y)$. Take $v, w \in R_{1}$ and $\alpha \in \Gamma$. Then

$$
\begin{aligned}
& \text { 1. }\left(\phi^{-1}\left(\lambda_{\gamma_{2}}\right)\right)_{x}(v+w)=\lambda_{\gamma_{2 \psi(x)}}(\phi(v+w)) \\
& =\lambda_{\gamma_{2 \psi(x)}}(\phi(v)+\phi(w)) \\
& \geq \min \left\{\lambda_{\gamma_{2 \psi(x)}}(\phi(v)), \lambda_{\gamma_{2 \psi(x)}}(\phi(w))\right\} \\
& =\min \left\{\phi^{-1}\left(\lambda_{\gamma_{2}}\right)_{x}(v), \phi^{-1}\left(\lambda_{\gamma_{2}}\right)_{x}(w)\right\} \\
& 2 .\left(\phi^{-1}\left(\mu_{\gamma_{2}}\right)\right)_{x}(v+w)=\mu_{\gamma_{2 \psi(x)}}(\phi(v+w)) \\
& =\mu_{\gamma_{2 \psi(x)}}(\phi(v)+\phi(w)) \\
& \leq \max \left\{\mu_{\gamma_{2 \psi(x)}}(\phi(v)), \mu_{\gamma_{2 \psi(x)}}(\phi(w))\right\} \\
& =\max \left\{\phi^{-1}\left(\mu_{\gamma_{2}}\right)_{x}(v), \phi^{-1}\left(\mu_{\gamma_{2}}\right)_{x}(w)\right\} \\
& 3 .\left(\phi^{-1}\left(\delta_{\gamma_{2}}\right)\right)_{x}(v+w)=\delta_{\gamma_{2 \psi(x)}}(\phi(v+w)) \\
& =\delta_{\gamma_{2 \psi(x)}}(\phi(v)+\phi(w)) \\
& \leq \max \left\{\delta_{\gamma_{2 \psi(x)}}(\phi(v)), \delta_{\gamma_{2 \psi(x)}}(\phi(w))\right\} \\
& =\max \left\{\phi^{-1}\left(\delta_{\gamma_{2}}\right)_{x}(v), \phi^{-1}\left(\delta_{\gamma_{2}}\right)_{x}(w)\right\}
\end{aligned}
$$

$$
\begin{aligned}
& \text { 4. }\left(\phi^{-1}\left(\lambda_{\gamma_{2}}\right)\right)_{x}(v \alpha w)=\lambda_{\gamma_{2 \psi(x)}}(\phi(v \alpha w)) \\
& =\lambda_{\gamma_{2 \psi(x)}}(\phi(v) \alpha \phi(w)) \\
& \geq \min \left\{\lambda_{\gamma_{2 \psi(x)}} \phi(v), \lambda_{\gamma_{2 \psi(x)}} \phi(w)\right\} \\
& =\min \left\{\phi^{-1}\left(\lambda_{\gamma_{2}}\right)_{x}(v), \phi^{-1}\left(\lambda_{\gamma_{2}}\right)_{x}(w)\right\} \\
& 5 .\left(\phi^{-1}\left(\mu_{\gamma_{2}}\right)\right)_{x}(v \alpha w)=\mu_{\gamma_{2 \psi(x)}}(\phi(v \alpha w)) \\
& =\mu_{\gamma_{2 \psi(x)}}(\phi(v) \alpha \phi(w)) \\
& \leq \max \left\{\mu_{\gamma_{2 \psi(x)}} \phi(v), \mu_{\left.\gamma_{2 \psi(x}\right)} \phi(w)\right\} \\
& =\max \left\{\phi^{-1}\left(\mu_{\gamma_{2}}\right)_{x}(v), \phi^{-1}\left(\mu_{\gamma_{2}}\right)_{x}(w)\right\} \\
& 6 .\left(\phi^{-1}\left(\delta_{\gamma_{2}}\right)\right)_{x}(v \alpha w)=\delta_{\gamma_{2 \psi(x)}}(\phi(v \alpha w)) \\
& =\delta_{\gamma_{2 \psi(x)}(\phi)}(\phi(v) \alpha \phi(w)) \\
& \leq \max \left\{\delta_{\gamma_{2 \psi}(x)} \phi(v), \delta_{\gamma_{2 \psi(x)}} \phi(w)\right\} \\
& =\max \left\{\phi^{-1}\left(\delta_{\gamma_{2}}\right)_{x}(v), \phi^{-1}\left(\delta_{\gamma_{2}}\right)_{x}(w)\right\}
\end{aligned}
$$

Thus $\left(\phi^{-1}\left(\gamma_{2}\right)\right)_{x}$ is a tripolar fuzzy $\Gamma$-subsemiring of $R_{1}$ for all $x \in \phi^{-1}(Y)$. Therefore $\left(\left(\phi^{-1}\left(\gamma_{2}\right)\right),\left(\psi^{-1}(Y)\right)\right.$ is a tripolar fuzzy soft $\Gamma$-subsemiring of $\left(\gamma_{1}, X\right)$ over $R_{1}$.

\section{Conclusion}

In this paper, we studied the concept of tripolar fuzzy soft $\Gamma$ - semiring homomorphism and discussed some properties of homomorphic image and pre image of tripolar fuzzy soft $\Gamma$-semiring. These concepts are basic supporting structures for development the theory of soft set. This work can be extended to the properties of different notions of kernel of tripolar fuzzy soft $\Gamma$ - semiring homomorphism, tripolar fuzzy soft filters over $\Gamma$ - semirings and tripolar fuzzy soft prime and maximal ideals.

Acknowledgements: Authors are thankful to the referee for there valuable suggestions.

\section{References:}

[1] H.S. vandiver., Note on a simple type of algebra in which cancellation law of addition does not hold, Ball. Amer. Math. soc. (N.S), 40(1934) 914-920.

[2] N.Nobusawa., On a generlization of the ring theory, Osaka. J. Math, 1(1964) 81-89.

[3] M. K. Sen., On $\Gamma-$ Semigroup, proc. of internatinal conference of algebra and its application, (1981), Decker publication, newyork, 301-308.

[4] M. Murali Krishna Rao., $\Gamma$-Semirings- $I$, Southeast Asian Bull. Math, 19(1) (1995) 49 54.

[5] L. A. Zadeh., Fuzzy sets, inform and control, 8 (1965) 338-353. 
[6] A. Rosenfeld., Fuzzy groups, j. Math. Anal. Appl., 35 (1971) 512-517.

[7] N. Ajmal and I. jahan, A study of normal fuzzy subgroups and characteristic fuzzy subgroups of a fuzzy group, Fuzzy information and engineering 4(2) (2012) 1-9

[8] Y. li, X. wang and L. Yang, A study of $(\lambda, \mu)$-fuzzy subgroups, Journal Applied mathematics, volume (2013) 1-7.

[9] M. O. Massa'deh., P and P* upper fuzzy subgrpups, Far East Journal of Mathematical Science, 5(2) (2010) $97-104$.

[10] M. O. Massa'deh., short communication, Some properties of upper fuzzy order, African Journal of Mathematical and computer science research, 3(2010) $192-194$.

[11] M. O. Massa'deh., Some structure properties of Anti L-Q-fuzzy and normal fuzzy subgroups, Asian Journal of Algebra, 5(1) (2012) 21-27.

[12] M. O. Massa'deh., On fuzzy subgroups with operator, Asian Journal of Mathematical and statistics, 5(4) (2012) 163 -166.

[13] D. Molodtsov., Soft set theory- first results, Computers and Mathetatics with Application, 37(1999) 19-31.

[14] P. K. Maji., R. Biswas and A. R. Roy., Fuzzy soft Sets, J. of Fuzzy Math., 9(2001) pp. 589 - 602.

[15] J. Ghosh., B. Dinda. and T.K. Samanta., Fuzzy soft rings and Fuzzy soft ideals,. Inter. J. of pure and appl. sci. and techn., 2 (2011) $66-74$.

[16] M. Mnrali Krishna Rao., Fuzzy soft ideal, fuzzy soft bi-ideal, fuzzy soft quasi-ideal and fuzzy soft interior ideal over ordered $\Gamma$-Semiring, Asia. Pac. J. Math., 5(2018) pp. 60-81.

[17] M. A. öztürk and E. Inan, Fuzzy soft subnearrings and $(\epsilon, \epsilon v q)$-fuzzy soft subnear-rings, computers of math. with Appli., 63 (3)(2012) 617628.

[18] K. T. Atanssov, Intuitionistic fuzzy sets, Theory and applications, Physica-Vertay, New York, 1999.

[19] M.O. Massa'deh., Structure properties of an Intuitionistic anti fuzzy M-subgroups, Journal of Applied computer science and Mathematics, 7(14), (2013), 42 -44.
[20] M.O. Massa'deh., A study on Intuitionistic fuzzy and normal fuzzy M-subgroup, MHomomorphism and Isomomorphism, International journal industrial Mathematics, 8(3), (2015), 185-188.

[21] M.O. Massa'deh. and T. Al-Hawary, Homomorphism in t-Q-intuitionistic L-fuzzy subrings, International journal of pure and applied Mathematics, 106(4), (2016), 1115-1126.

[22] M.O. Massa'deh., Some contribution on Intuitionistic Q-fuzzy Ku-ideals, JP journal of Algebra, Number Theory and Applications 42(1), (2019), 95-110.

[23] M.O. Massa'deh. and A. Fellatah, Some properties on Intuitionistic Q-fuzzy K-ideals and KQ-fuzzy ideals in $\Gamma$-semirings, Africa Matematika, 31, (2019), 1145-1152.

[24] P. K. Maji., R. Biswas and A. R. Roy, On Intuitionistic fuzzy soft sets, Journal of Fuzzy Mathematic, 9(3), (2001), 667-692.

[25] N.Yaqoob, M. Akram. and M. Aslam., Intuitionistic Fuzzy Soft Groups include by $(t, s)$-norm, Indian Journal of Science and Technology, 6(4), (2013), 4283-4289.

[26] W. R. Zhang., Bipolar fuzzy sets, Proc. of fuzzy IEEE (1998) pp. 835-840.

[27] K.M. Lee., Bipolar-valued fuzzy sets and their operations, Proc. international conference on intelligent Technologies, Bangkok, Thailand, (2000), 307-312.

[28] M.O. Massa'deh. and F. Ismail, Bipolar $Q$-Fuzzy $H$-ideals over $\Gamma$-Hemiring, Advance in fuzzy Mathematics, 13(1), (2018), 15 24.

[29] M.O. Massa'deh., A Study on Anti Bipolar $Q$-Fuzzy Normal semigroup's, Journal of Mathematical sciences and Applications, 6(1), (2018), 1-6.

[30] M.O. Massa'deh., On Bipolar fuzzy cosets, Bipolar fuzzy ideals and homomorphism of $\Gamma$-nearrings, Far East Journal of Mathematical of Science, 102(4) , (2017), 163-178.

[31] M. Akram., Bipolar fuzzy soft lie algebras, Quasigroups and related systems, 21, (2013), 110. 
[32] M. Mnrali Krishna Rao., Tripolar fuzzy interior ideal of $\Gamma$-Semigroup, Anl. of fuzzy Math. and info., 15, (2018), 199-206.

[33] M. Mnrali Krishna Rao , B.Venkateswarulu. and Y. Adi Narayana, Tripolar fuzzy soft ideals and tripolar fuzzy soft interior ideals over semiring, Italian journal of pure and applied Mathematics, 42, (2019), 731-743. 